ZOOLOGIA 30 (3): 338-342, June, 2013

http://dx.doi.org/10.1590/S1984-46702013000300013

\title{
A new species and new records of linyphiid spiders (Araneae: Linyphiidae) from Amazonas, Brazil
}

\section{Everton Nei Lopes Rodrigues', Yuji Lemos² \& Antonio Domingos Brescovit ${ }^{2}$}

\author{
1 Programa de Pós-Graduação em Biologia, Universidade do Vale dos Sinos. Avenida Unisinos 950, Cristo Rei, \\ 93022-000 São Leopoldo, RS, Brazil.E-mail: enlrodrigues@yahoo.com.br \\ ${ }^{2}$ Laboratório Especial de Coleções Zoológicas, Instituto Butantan. Avenida Vital Brazil 1500, 05503-900 São Paulo, SP, \\ Brazil.E-mail: yujilemos@gmail.com; adbresc@terra.com.br
}

\begin{abstract}
Linyphiidae is currently the second richest family of spiders in the order Araneae but is poorly known and studied in Brazil, particularly in the northern states. In this contribution we describe a new species of Exechopsis Millidge, 1991 from the State of Amazonas, based on both males and females. The male of Meioneta adami Millidge, 1991 is described for the first time. Mermessus conjunctus (Millidge, 1991), Novafrontina uncata (F. O. Pickard-Cambridge, 1902), Sphecozone fastibilis (Keyserling, 1886), Sphecozone ignigena (Keyserling, 1886) and Vesicapalpus simplex Millidge, 1991 are recorded for the first time from Amazonas. Sphecozone crassa (Millidge, 1991) are presented additional record from Amazonas.
\end{abstract}

KEY WORDS. North of Brazil; Neotropical Region; complementary descriptions; spider taxonomy.

The Linyphiidae consists mainly of very small spiders, which are sheetweb builders (RoberTs 1987, Draney \& Buckle 2005, Miller 2007). The presence of a discrete, intersegmental paracymbium (rarely missing); stridulatory striae file on the ectal side of the chelicerae; and autospasy of the legs at the patella-tibia junction distinguish this family from all others, except Pimoidae (Draney \& Buckle 2005). Linyphiids distinguished from Pimoidae by lacking a retrolateral cymbial sclerites attached to the cymbium via a membrane (Draney \& BUCKLE 2005).Linyphiidae, with 4,412 extant species in 587 genera (Platnick 2012) is the second richest family of spiders in the order Araneae in terms of number of species. Despite of this, taxonomic studies on Neotropical Linyphiidae are rare.

Most species of Linyphiidae were described from South America by Millidge (1991), and only two species are known to occur in the state of Amazonas, in the northern portion of Brazil - Meioneta adami Millidge, 1991 and Tutaibo niger (O. PickardCambridge, 1882).

The examination of specimens from State of Amazonas, in Brazilian collections, allowed the recognition and description of a new species of Exechopsis Millidge, 1991, based both males and females, as well as the description of the male of Meioneta adami Millidge, 1991 for the first time. The following species are recorded for the first time from Amazonas: Mermessus conjunctus (Millidge, 1991), Novafrontina uncata (F.O. PickardCambridge, 1902), Sphecozone fastibilis (Keyserling, 1886), Sphecozone ignigena (Keyserling, 1886) and Vesicapalpus simplex
Millidge, 1991. Additional records of Sphecozone crassa (Millidge, 1991) are presented.

\section{MATERIAL AND METHODS}

All specimens examined and studied herein are deposited in the arachnological collections of the Instituto Butantan, São Paulo (IBSP, D.M.B. Battesti, curator); Instituto Nacional de Pesquisas da Amazônia, Manaus (INPA, C. Magalhães) and Museu de Ciências Naturais da Fundação Zoobotânica do Rio Grande do Sul, Porto Alegre (MCN, R. Ott). For illustrations, the specimens were examined under a Leica ${ }^{\circledR}$ MZ9.5 with camera lucida. The study of the reproductive structures of both males and females was performed by immersing the epigynum and the embolic division in methyl salicylate and/or clove oil for approximately 30 minutes until the internal structures could be perfectly visualized. The palp was immersed in $10 \%$ potassium hydroxide for approximately two hours, and after that immersed in distilled water (Levi 1965). This process expands it and allows for a better visualisation of the parts. The specimens are preserved in 70-80\% ethanol. All measurements were made using a scale reticle on the objective of the stereomicroscope, and are expressed in millimeters. The descriptions and terminology follow Millidge (1980) and Miller (2007), respectively. The tibial spine formula follows RoвERTs (1987). Abbreviations: (AME) anterior median eyes, (ALE) anterior lateral eyes, (PLE) posterior lateral eyes and (PME) posterior median eyes. 


\section{TAXONOMY}

\section{Exechopsis eberhardi sp. nov. \\ Figs 1-8}

Types. Holotype male from km 41, BR 174, Reserva 1501 INPA, ZF3, Manaus, Amazonas, W.G. Eberhard leg., deposited in the INPA 1875. Paratype female, same data as the holotype, deposited in the INPA 8596. Paratypes four males and five females from Reserva do PDBFF, Manaus, Amazonas, II-IX.2001; E. M.Venticinque leg., deposited in the IBSP 55541, 55546, 55571.

Diagnosis. The male of Exechopsis eberhardi sp. nov. resembles that of E. versicolor Millidge, 1991 (Millidge 1991: 56, figs $184,185,188$ ) in the shape of the tegulum and subtegulum; the radix developed (Fig. 2) and large eyes with AME protuberant (Fig. 5). It differs from it in the radix mesally rounded near the margin of cymbium (Fig. 2), tegulum wider distally and lamella characteristica with blunt end (Figs 1 and 2). The female of E. eberhardi sp. nov. is characterized by epigynum with a relatively large atrium, without median septum; ventral plate wider than long and dorsal plate developed, wider in the posterior region (Fig. 7).

Description. Male (INPA 1875, holotype): Total length 1.40. Carapace length 0.65 , width 0.50 . Clypeus height 0.08 . Sternum length 0.33 , width 0.35 . Chelicerae length 0.33 . Abdomen length 0.69 , width 0.48 , height 0.52 . Leg lengths (I/II/ III/IV): femora $0.81 / 0.75 / 0.46 / 0.63$; patellae $0.21 / 0.21 / 0.14$ / 0.18 ; tibiae $0.69 / 0.63 / 0.33 / 0.48$; metatarsi $0.84 / 0.65 / 0.44 / 0.63$; tarsi 0.48/-/0.33/0.42; total 3.03/-/1.70/2.32. Palp (femur, patella, tibia, cymbium): 0.18/0.12/0.08/0.23. Coxa IV basis well apart from each other (0.12). Metatarsal trichobothria I-III present, IV absent. Tibial spine formula: 1-1-1-1. Eye diameters and interdistances: AME and ALE 0.08, PME and PLE 0.06; AMEALE 0.03, PME-PLE 0.04, AME-AME 0.02, PME-PME 0.05. Eyes (Figs 3 and 5) margined with black; AME projected. Clypeus glabrous. Carapace yellow with median and lateral dark-brown dots, longer than wide, setae present at ocular area. Chelicerae yellow with setae. Labium brown. Endites yellow with setae. Sternum yellow with darkened borders and sparse setae covering. Chelicerae promargin with four teeth, retromargin with three teeth. Coxae yellow, except coxae IV with dark-brown dots; femur yellow, other segments yellow covered with darkbrown dots. Abdomen longer than wide (Fig. 3), dorsally yellow, posteriorly dark-brown bearing small white-perolized spots and a lateral (Fig. 4) white-perolized band; venter brown. Spinnerets and colulus brown. Colulus well developed. Palpal paracymbium U-shaped, less developed (Fig. 1). Embolic membrane present (Fig. 2), tegulum with sinuous duct (Fig. 1) and long embolus (Fig. 2). Subtegulum large (Fig. 1). Radix developed. Suprategulum sclerotized (Fig. 2). Retrobasal cymbial groove sclerotized. Anterior radical process (Figs 1 and 2) flagelliform, with apex near the embolus.

Female (Paratype, INPA 8596): total length 1.84. Carapace length 0.75 , width 0.56 . Clypeus height 0.08 . Sternum length 0.39 , width 0.42 . Chelicerae length 0.39 . Abdomen length 1.11 , width 0.88 , height 0.79 . Leg formula I/II/IV/III; lengths (I/II/III/IV): femora 0.92/0.88/0.56/0.81; patellae 0.23 / $0.23 / 0.16 / 0.21$; tibiae $0.75 / 0.69 / 0.42 / 0.60$; metatarsi $0.84 / 0.79 /$ 0.44/0.77; tarsi 0.50/0.50/0.35/0.39; total 3.24/3.09/1.93/2.78. Palp (Fig. 6; femur, patella, tibia, tarsi): 0.23/0.10/0.14/0.29. Coxa IV basis well appart from each other (0.18). Metatarsus IIII with trichobothrium, IV absent. Tibial spine formula: 1-1-11. Eye diameters and interdistances: AME 0.09, ALE 0.08, PME 0.08 and PLE 0.08; AME-ALE 0.03, PME-PLE 0.04, AME-AME 0.02, PME-PME 0.04. Eyes (Fig. 6) margined with black; AME projected. Clypeus glabrous. Carapace yellow with median and lateral dark-brown spots, longer than wide, setae present at ocular area. Chelicerae pale orange with setae. Labium brown. Endites pale brown with setae. Sternum with setae, pale brown, borders dark. Chelicerae promargin and retromargin with five teeth. Coxae pale yellow, except coxae IV with dark-brown dots; femur pale yellow, other segments yellow covered with darkbrown dots. Abdomen longer than wide (Fig. 8), dorsally brown with median area not pigmented, posteriorly dark-brown bearing white-perolized band and lateral with white-perolized band; venter brown. Spinnerets and colulus brown. Colulus well developed. Epigynum with prominent ventral plate, large, wide than longer (Fig. 7). Dorsal plate prominent (Fig. 7).

Distribution. Brazil (Amazonas).

Etymology. The specific name honors the arachnologist William G. Eberhard, collector of the type specimens.

\section{Meioneta adami Millidge, 1991}

Figs 9-11

Meioneta adami Millidge, 1991: 105, fig. 420 (Holotype female from Manaus, Amazonas, February 1977, N.A. Locket leg., deposited in the AMNH, not examined).

Diagnosis. The palp of Meioneta adami resembles that of M. straminicola Milligde, 1991 (Millidge 1991: figs 388-390) in having the mesal apophysis in cymbium (Fig. 10, apophysis IV) and embolus pointed distally (Figs 9 and 10), but differs in the ventrally elongated membranous lamella characteristica (Fig. 9); presence of four basal apophyses in cymbium (Figs 9 and 10); and the dorsal view of male palpal tibia (Fig. 11).

Description. Male (IBSP 120214): total length 1.26. Carapace length 0.67 , width 0.54 . Clypeus height 0.12 . Sternum length 0.35 , width 0.35 . Chelicerae length 0.30 . Abdomen length 0.58 , width 0.35 , height 0.31 . Leg formula I/IV/II/III; segment lengths (I/II/III/IV): femora 0.75/0.60/0.46/0.63; patellae $0.16 /$ $0.18 / 0.16 / 0.16$; tibiae $0.69 / 0.58 / 0.42 / 0.67$; metatarsi $0.60 / 0.46 /$ 0.42/0.58; tarsi 0.39/0.39/0.33/0.35. Total 2.59/2.21/1.79/2.39. Palp (femur, patella, tibia, cymbium): 0.35/0.10/0.12/0.38. Coxa IV bases well separated (0.10). Metatarsal trichobothria I-IV present. TmI 0.35. Tibial spine formula: 2-2-2-2. Eye diameters and interdistances: AME 0.03, PME 0.05, ALE and PLE 0.04; AMEALE 0.04, PME-PLE 0.05, AME-AME 0.02, PME-PME 0.03. Eyes margined with black. Carapace longer than wide, brown with 

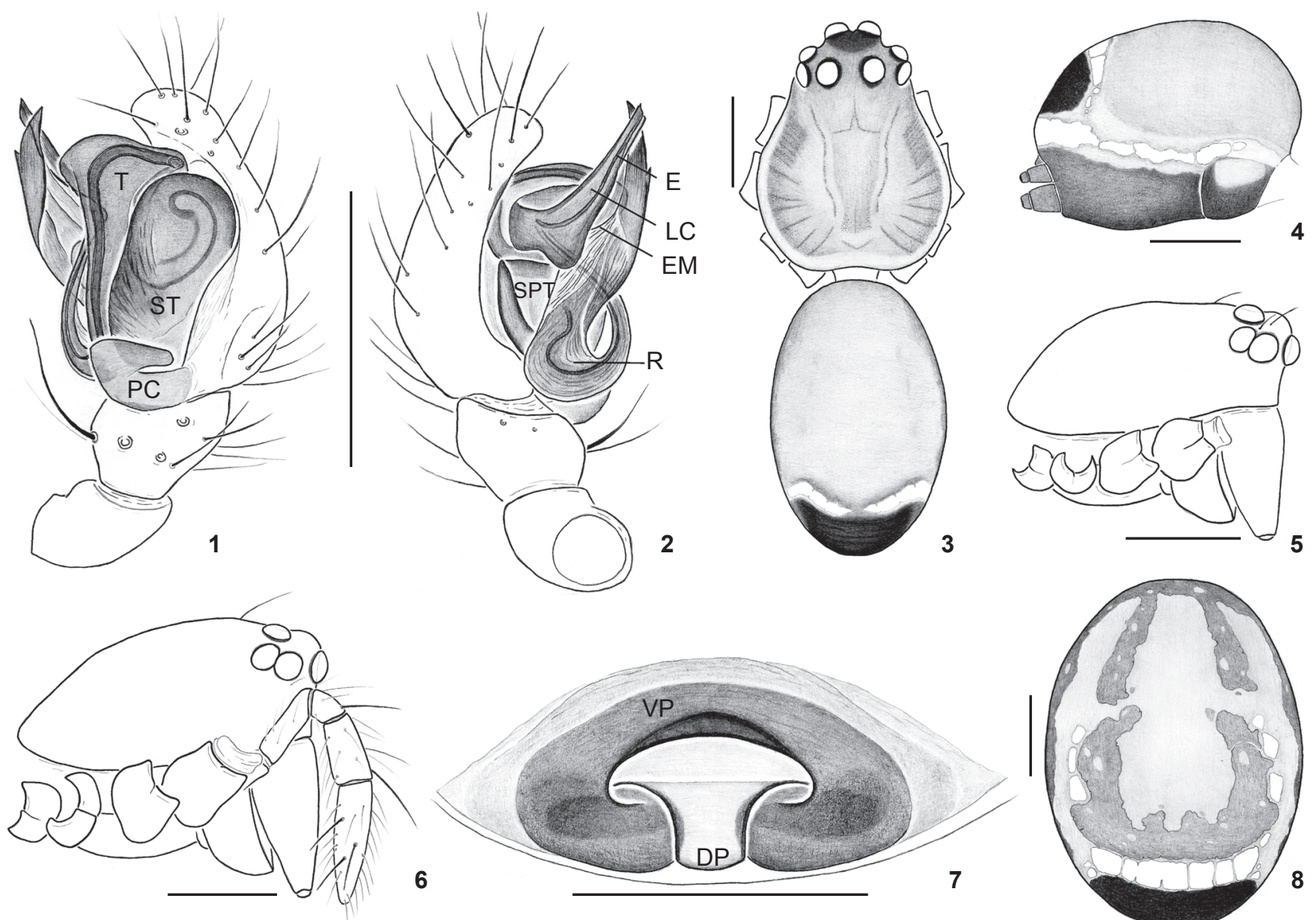

Figures 1-8. Exechopsis eberhardi sp. nov.: (1-2) Male palp: (1) ectal, (2) mesal. (3-5) Male: (3) habitus, dorsal, (4) abdomen, lateral; (5) carapace, lateral. (6-8) Female: (6) carapace; lateral; (7) epigyne, ventral; (8) abdomen, dorsal. (DP): dorsal plate, (E) embolus, (EM) embolic membrane, (LC) lamella characteristica, (PC) paracymbium, (R) radix, (SPT) suprategulum, (ST) subtegulum, (T) tegulum, (VP) ventral plate, (I-IV) apophysis is cymbium. Scale bars: $1,2,7=0.25 \mathrm{~mm}, 3-5,8=0.50 \mathrm{~mm}$.

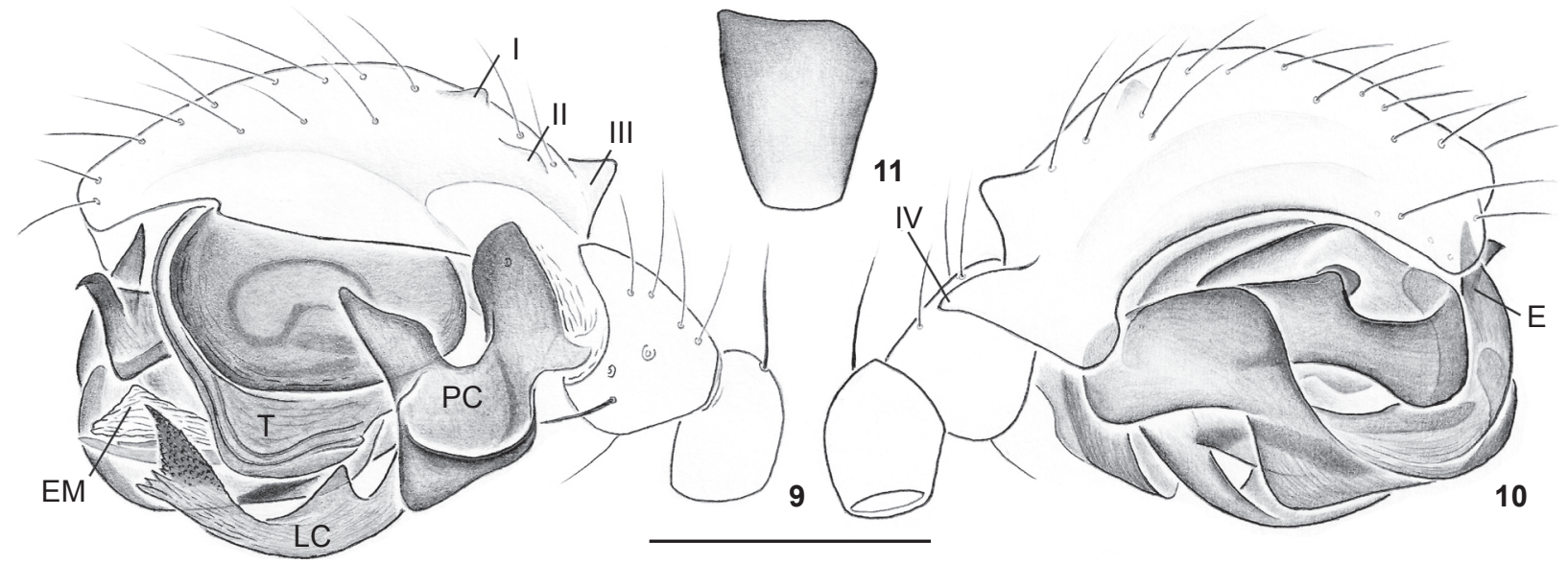

Figures 9-11. Meioneta adami: (9-10) male palp: (9) ectal; (10) mesal; (11) male palpal tibia, dorsal. Scale bars: $0.25 \mathrm{~mm}$. 
dark-brown dots. Chelicerae and endites brown. Labium dark brown. Sternum brown, with borders dark-brown. Chelicerae retromargin with three small teeth, promargin with three teeth. Legs yellow, covered with dark brown dots (especially on femur); coxa pale yellow with dark-brown dots posteriorly. Abdomen longer than wide, dorsally yellow, anteriorly and posteriorly brown; ventrally dark-brown. Spinnerets and colulus brown with dark brown dots. Palp: dorsal surface of cymbium with tiny apophysis (Fig. 9); cymbium, as viewed mesally, with two basal projections (Fig. 10). Paracymbium large (Fig. 9). Embolus with curved distal portion ending near apex of the cymbium, embolic membrane present (Figs 9 and 10). Tegulum with sinuous margin and light duct, which forms a sinuous curve (Fig. 9). Lamella characteristica membranous, longer than wide (Fig. 9).

Female. Described by Millidge (1991: 105).

Material examined. Brazil, Amazonas: Manaus (Lago Janauari; 0320'S; 60¹7'W), 15.X.1987, J. Adis et al. leg., 1 female (IBSP 120159); VI.1987-VII.1988, 2 males and nine females (IBSP 120152, 120159, 120180, 120188, 120190, 120210, 120214, 120235).

Distribution. Brazil (Amazonas).

Remark. Males and females were collected together in Amazonas, Brazil.

\section{Mermessus conjunctus (Millidge, 1991)}

Eperigone conjuncta Millidge, 1991: 141, figs 573-575 (holotype female from 5 km east of Belém, Pará, Brazil, May 2, 1974, R.T. Schuh leg., deposited in the AMNH, not examined).

Mermessus conjunctus, Miller, 2007: 123 (transfered from Eperigone); Platnick, 2012.

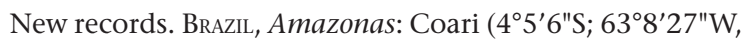
Base de Operações Geólogo Pedro de Moura, Porto Urucu), 1 female, XI.2006, S.C. Dias leg. (MCN 44819).

Distribution. Brazil (Amazonas and Pará).

\section{Novafrontina uncata (F.O. Pickard-Cambridge, 1902)}

Frontinella uncata F.O. Pickard-Cambridge, 1902: 422, pl. 40, figs 5-6 (Male and female syntypes from Guatemala, deposited in the BMNH, not examined).

Linyphia uncata, Petrunkevitch, 1911: 255; Roewer, 1942: 591; Bonnet, 1957: 2537.

Novafrontina uncata, Millidge, 1991: 66, figs 216-217, 219, 221 222, 224 (transfered from Linyphia); Millidge, 1993: 146, fig. 14; Platnick, 2012.

New records. BRAZIL, Amazonas: Manaus (Reserva Florestal Adolpho Ducke, 257’42"S; 5955’40"W), 1 male, 03.VIII.1987, J. Vital leg. (INPA 2313); 1 male, two females, 18.XII.1987, A.A. Lise leg. (INPA 2314); 3 females, 18.XII.1987, A.A. Lise leg. (INPA 2314); São Gabriel da Cachoeira (Maturacá, 0³7'28"N; $\left.66^{\circ} 7^{\prime} 41^{\prime \prime} \mathrm{W}\right), 1$ female, 11.X.1990, A.A. Lise leg. (INPA2317); 2 females (INPA 2311); 1 female (INPA 2315); one female, 18.XII.1987,
J.A. Vidal leg. (MCN 18255); 1 female, 18.XII. 1987, A.A. Lise leg. (MCN 18256); 1 female, 18.XII.1987, A.A. Lise leg. (MCN 18257). Distribution. Brazil (Amazonas and Pará), Colombia (Meta, Valle), Guatemala, Mexico, Panama and Venezuela (Amazonas).

\section{Sphecozone fastibilis (Keyserling, 1886)}

Erigone fastibilis Keyserling, 1886: 217, pl. 19, fig. 274 (Holotype female from Serre Vermelle [sic, Serra Vermelha], Minas Gerais, Brazil, deposited in the BMNH, not examined); Keyserling, 1891: 243. Göldi, 1892: 225.

Clitolyna fastibilis, Simon, 1894: 673. Petrunkevitch, 1911: 227. Roewer, 1942: 708. Bonnet, 1956: 1101.

Clitolina fastibilis, Petrunkevitch, 1928: 129.

Sphecozone fastibilis, Miller, 2007: 208, figs 154A-E, 164 (transfered from Clitolyna). Platnick, 2012.

New record. BraziL, Amazonas: Manaus (Fazenda Esteio, $02^{\circ} 24^{\prime} \mathrm{S} ; 59^{\circ} 52^{\prime} \mathrm{W}, 60 \mathrm{~km} \mathrm{~N}$ Manaus, ZF 3, Km 23), 1 female, 02.X.1985 (INPA 2323); 1 male, 26.III.1986 (INPA 2324); 1 male, 02.X.1985 (INPA 2325); 1 male, 10.VII.1985 (INPA 2326) all coleted by B.C. Klein.

Distribution. Brazil (Amazonas, Minas Gerais and Rio Grande do Sul) and Argentina (Misiones).

\section{Sphecozone ignigena (Keyserling, 1886)}

Erigone ignigena Keyserling, 1886: 268, fig. 316 (Holotype male from Rio Grande, Brazil, deposited in The Natural History Museum, London (BMNH), not examined). Keyserling, 1891: 243. Petrunkevitch, 1911: 235. Roewer, 1954: 1498. Bonnet, 1956: 1765 .

Sphecozone tincta Millidge, 1991: 174, figs 731, 732, 737 (Holotype male from Pelotas, State of Rio Grande do Sul, Brazil, November, 1960, C. Biezanko leg., deposited in the American Museum of Natural History, New York (AMNH), not examined). Platnick, 1993: 354. Rodrigues, 2005: 103, figs 111 (description of female). Miller, 2007: 198 (syn.).

Sphecozone ignigena, Miller, 2007: 198, figs 143, 148A-B, G, 151B, 164 (transfered from Erigone). Platnick, 2012.

New record. BraziL, Amazonas: Manaus (Ilha da Marchantaria, Rio Solimões, $3^{\circ} 16^{\prime} 7.60^{\prime \prime} \mathrm{S} ; 5^{\circ} 59^{\prime} 57.86^{\prime \prime} \mathrm{W}$ and $3^{\circ} 13^{\prime} 1.60^{\prime \prime} \mathrm{S}$; 5953'59.68"W), 6 males, 04.XI.1981, J. Adis leg. (MCN 22008).

Distribution. Brazil (Amazonas and Rio Grande do Sul) and Argentina (Entre Ríos).

\section{Sphecozone crassa (Millidge, 1991)}

Gymnocymbium crassum Millidge, 1991: 184, figs 800-802 (Holotype male from Sinop, Mato Grosso, Brazil, Oct. 1975, M. Alvarenga leg., deposited in the AMNH, not examined). Platnick, 1993: 283.

Gymnocymbium propinquum Millidge, 1991: 186, figs 804, 807 (Holotype female from Jacazeacanga (sic, Jacareacanga), Pará, Brazil, October 1959, M. Alvarenga leg., deposited in the AMNH, not examined). Platnick, 1993: 283. 
Sphecozone crassa, Miller, 2007: 205, figs 151E, 153A, B, E, G, H, 162A-F, 163A-F, 165 (transfered from Gymnocymbium).

Additional records. BRAzIL, Amazonas: Manaus (Reserva do

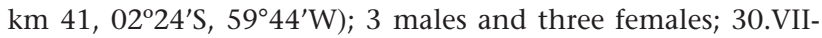
02.IX.2008; C.M.P. Leite leg. (IBSP 121056); Presidente Figueiredo (Reserva Biológica Uatumã, $\left.1^{\circ} 48^{\prime} 58.7^{\prime \prime} S, 59^{\circ} 15^{\prime} 16.6^{\prime \prime} \mathrm{W}\right), 2$ males, 01.X.2006, J.T. Nascimento leg. (INPA 0710); 2 males, 1 female (INPA 0711); 2 males, two females (INPA 0712); 1 female (INPA 2322); 2 males, 1 female (INPA 2548); 1 male, one female (INPA 2694); Coari ( $4^{\circ} 5^{\prime} 6^{\prime \prime} \mathrm{S}, 6^{\circ} 8^{\prime} 27^{\prime \prime} \mathrm{W}$, Base de Operações Geólogo Pedro de Moura, Porto Urucu), 2 females, 26.X.2006, D.F. Candiani leg. (MCN 44819).

Distribution. Brazil (Amazonas, Pará and Mato Grosso) and Colombia (Caquetá).

\section{Vesicapalpus simplex Millidge, 1991}

Vesicapalpus simplex Millidge, 1991: 56, figs 182, 183 (Holotype male, Tobunas, Misiones, Argentina, VII-VIII.1959, O de'Ferrarris leg., deposited in the AMNH, not examined); Rodrigues \& Ott, 2006: 410, figs 10-16.

New record. BRAZIL. Amazonas: Manuas (Reserva Florestal Adolpho Ducke, $2^{\circ} 57^{\prime} 42^{\prime \prime}$ S; 5955'40"W), 1 male, 04.VIII.1987, A.A. Lise leg. (INPA 2316).

Distribution. Brazil (Amazonas and Rio Grande do Sul) and Argentina (Misiones).

\section{ACKNOWLEDGEMENTS}

We thank the curators of the arachnological collections for loaning material and Lidianne Salvatierra (INPA) for information provided. To the director's office of the Museu de Ciências Naturais (MCN, FZB-RS) for allowing the senior author to carry out part of this work in its facilities. Everton N.L. Rodrigues was supported by FAPESP (process 2011/02036-7). Antonio D. Brescovit is supported by CNPq (process 301776/2004-0) and Yuji Lemos is supported by Capes. We also thank two anonymous reviewers and the editor for their valuable comments.

\section{LITERATURED CITED}

Draney, M.L. \& D.J. Buckle. 2005. Chapter 35: Linyphiidae, p.124-161. In: D.UBick; P. PAQUin; P.E. Cushing \& V. Roth (Eds). Spiders of North America: an identification manual. American Arachnological Society.

GöLDI, E.A. 1892. Zur Orientierung in der Spinnenfauna Brasiliens. Mitteilungen aus dem Osterlande 5: 200-248.

BONNET, P. 1956. Bibliographia araneorum; analyse méthodique de toute la littérature araneologique jusqu'en 1939. Toulouse, Douladore, vol. 2, part 2, p. 919-1926.

BONNET, P. 1957. Bibliographia araneorum; analyse méthodique de toute la littérature araneologique jusqu'en 1939. Toulouse, Douladore, vol. 2, part 3, p. 1927-3026.
Keyserling, E. 1886. Die Spinnen Amerikas, Theridiidae. Nürnberg, Bauer \& Raspe, Bd. 2, Hälfte 2, 295p.

Keyserling, E. 1891. Die Spinnen Amerikas: Brasilianische Spinnen. Nürnberg, vol. 3.

Levi, H.W. 1965. Techniques for the study of spider genitalia. Psyche 72: 152-158.

Miller, J.A. 2007. Review of erigonine spider genera in the Neotropics (Araneae: Linyphiidae, Erigoninae). Zoological Journal of the Linnean Society 149 (Suppl. 1): 1-263.

Millidge, A.F. 1980. The erigonine spiders of North America. Part 1. Introduction and taxonomic backgraund (Araneae: Linyphiidae). Journal of Arachnology 8: 97-107.

Millidge, A.F. 1991. Further linyphiid spiders (Araneae) from South America. Bulletin of the American Museum of Natural History 205: 1-199.

Millidge, A.F. 1993. Further remarks on the taxonomy and relationships of the Linyphiidae, based on the epigynal duct confirmations and other characters (Araneae). Bulletin of the British Arachnological Society 9: 145-156.

Petrunkevitch, A. 1911. A synonymic index-catalogue of spiders of North, Central and South America with all adjacent islands, Greenland, Bermuda, West Indies, Terra del Fuego, Galapagos, etc. Bulletin of the American Museum of Natural History 29: 1-791.

Petrunkevitch, A. 1928. Systema aranearum. Transactions of the Connecticut Academy of Arts and Sciences 29: 1-270.

Pickard-CAmbridge, F.O. 1902. Arachnida - Araneida and Opiliones. Biologia Centrali-Americana, Zoology 2: 313-424.

PlatnICK, N.I. 1993. Advances in spider taxonomy 1988-1991. New York, New York Entomological Society.

Platnick, N.I. 2012. The world spider catalog, version 13.0. American Museum of Natural History, available on line at: http://research.amnh.org/entomology/spiders/catalog/ index.html [Accessed: 30/I/2012].

Rовевтs, M.J. 1987. The spiders of Great Britan and Ireland, vol. 2: Linyphiidae and check list. Harley Books, Colchester, 204p.

Rodrigues, E.N.L. 2005. Descrição da fêmea de Sphecozone tincta (Araneae, Linyphiidae) e novas ocorrências no Brasil. Iheringia, Série Zoologia 95 (1): 103-105.

Rodrigues, E.N.L. \& R. Otт. 2006. Sobre o gênero Vesicapalpus (Araneae, Linyphiidae) no sul do Brasil. Iheringia, Série Zoologia 96 (4): 407-412.

Roewer, C.F. 1942. Katalog der Araneae von 1758 bis 1940. Bremen, Natura, vol. 1.

Roewer, C.F. 1954. Katalog der Araneae von 1758 bis 1940. Bruxelles, Institut Royale des Sciences Naturelles, vol. 2.

SIMON, E. 1894. Histoire naturelle des araignées. Paris, vol. 1, $2^{\text {nd }}$ ed., p. 489-760.

Submitted: 28.IX.2012; Accepted: 16.I.2013. Editorial responsibility: Walter A.P. Boeger 